\title{
HIGHLIGHTS
}

GENETICS

\section{PTPRC mutation associated with response to anti-TNF therapy in rheumatoid arthritis}

Patients with rheumatoid arthritis (RA) who harbor a particular mutation at the protein tyrosine phosphatase receptor type $\mathrm{C}(P T P R C)$ gene locus have a better response to anti-tumor necrosis factor (TNF) therapy than individuals who lack it. This finding, from a multicenter analysis by Jing Cui and colleagues, might eventually be incorporated into a composite genetic prediction tool for identifying patients with RA who are likely to benefit from drugs that inhibit TNF activity. "In addition to tailoring therapy to the appropriate patient population, a biomarker of treatment response would provide insight into drug mechanism of action and potentially enhance design approaches for more efficient larger-scale clinical trials," suggest the authors.

The researchers included data from nine RA cohorts $(n=1,283)$, with the inclusion criteria for patients being active disease prior to infliximab, etanercept or adalimumab treatment $(n=625,502$ and 156, respectively) and available disease activity score (DAS28) data within 3-12 months of commencing therapy. The investigators tested $31 \mathrm{RA}$ risk alleles for correlation with response to anti-TNF therapy and only one single nucleotide polymorphism (SNP) in PTPRC (also known as CD45) was significantly associated with response in both univariate and multivariate models ( $P=0.0004$ and $P=0.0001$, respectively). Following anti-TNF therapy, possession of the SNP, termed rs10919563, correlated with a 'good response' according to EULAR response criteria (DAS28 <3.2). Furthermore, the change in DAS28 from baseline was also associated with the presence of rs10919563.

Despite strong statistical evidence that the PTPRC SNP is a reliable predictor of response to anti-TNF therapy, the researchers remain cautious about interpreting the results owing to the heterogeneity between the nine cohorts (e.g. study design, ascertainment criteria, duration of follow-up, population ancestry). "We need to replicate this finding in other collections to verify it is real, following which it will be important to fine-map and understand the functional consequences of the mutation," explains Robert Plenge, the study's lead investigator, who goes on to say that "unbiased genomewide association studies are required to identify additional mutations that influence response to therapy."

Rowan Higgs

Original article Cui, J. et al. PTPRC rheumatoid arthritis risk allele is also associated with response to anti-TNF therapy. Arthritis Rheum. doi:10.1002/art.27457 